\title{
Continuous-Wave Laser Action on Vibrational-Rotational Transitions of $\mathrm{CO}_{2}$
}

\author{
C. K. N. Patel \\ Bell Telephone Laboratories, Murray Hill, New Jersey
}

(Received 15 July 1964)

\begin{abstract}
We have obtained $\mathrm{cw}$ laser action on a number of rotational transitions of the $\Sigma_{u}^{+}-\Sigma_{\theta}{ }^{+}$vibrational band of $\mathrm{CO}_{2}$ around 10.4 and $9.4 \mu$. The laser wavelengths are identified as the $P$-branch rotational transitions from $P(12)$ to $P(38)$ for the $00^{\circ} 1-10^{\circ} 0$ band and from $P(22)$ to $P(34)$ for the $00^{\circ} 1-02^{\circ} 0$ band. Strongest laser transition occurs at $10.6324 \mu$ (vacuum). A cw power output of about $1 \mathrm{~mW}$ has been measured. All these laser transitions can also be made to oscillate under pulsed discharge conditions with a small increase in the peak laser power output. No $R$-branch transitions have been seen to oscillate either under cw or pulsed discharge conditions. The wavelength measurements are in reasonable agreement with earlier measurement of the bands in absorption, but there are slight differences. These are ascribed to possible pressure-dependent frequency shift effects. A study has been made of the time dependence of the laser output under pulsed excitation, and some conclusions about possible excitation processes are given. Theoretical interpretation given earlier for laser action on vibrational-rotational transitions is discussed in a generalized form. The theory is applicable to both the linear polyatomic molecules and the diatomic molecules.
\end{abstract}

\section{INTRODUCTION}

$T$ $\mathrm{HE}$ vibrational-rotational bands of $\mathrm{CO}_{2}$ in the region of $1-15 \mu$ have been the subject of several investigations in the past. In particular the $\Sigma_{u}{ }_{-} \Sigma_{g}+$ $00^{\circ} 1-10^{\circ} 0$ and $00^{\circ} 1-02^{\circ} 0$ bands have been observed in absorption in laboratory ${ }^{1}$ as well as in absorption in the upper atmosphere. ${ }^{2}$ The $00^{\circ} 1-10^{\circ} 0$ band centered at $10.41 \mu$, and the $00^{\circ} 1-02^{\circ} 0$ band centered at $9.40 \mu$ have been investigated under high resolution by Barker and Adel. ${ }^{\prime}$ Since the lower terminal states $00^{\circ} 1$ and $02^{\circ} 0$ are situated about 1388 and $1286 \mathrm{~cm}^{-1}$, respectively, above the ground state of $\mathrm{CO}_{2}$ in order to achieve measurable absorption in the infrared region of 9.4 and $10.4-\mu$ bands, the $\mathrm{CO}_{2}$ pressure in the absorption cell has been quite high. (Typically atmospheric pressures together with absorption lengths at several meters have been used.) At these pressures, there is a likelihood of collisions causing shifts in transition frequencies and broadening of the individual lines. Observation of these bands in absorption in the upper atmosphere suffers owing to overlapping absorption bands of other constituents of the atmosphere, chiefly $\mathrm{H}_{2} \mathrm{O}$ and $\mathrm{O}_{3}$.

This paper reports the observation of $\mathrm{cw}$ optical maser action on the $P$-branch rotational transitions of the $00^{\circ} 1-10^{\circ} \mathrm{O}$ and $00^{\circ} 1-02^{\circ} 0$ vibrational bands of $\mathrm{CO}_{2}$ in a gaseous discharge. ${ }^{3}$ Previously, cw optical maser action has been reported on a number of atomic transitions of both the neutral atoms and the ionized atoms. The conclusions reached earlier ${ }^{4}$ about the possibility of improving wavelength determination of the laser transitions due to the brightness and coherence characteristic of laser output apply here also. Here the advantages of molecular vibrational-rotational transi-

\footnotetext{
${ }^{1}$ E. F. Barker and A. Adel, Phys. Rev. 44, 185 (1933).

${ }^{2}$ A. Adel, Astrophys. J. 94, 375 (1941).

${ }^{3} \mathrm{~A}$ preliminary report of the laser action in $\mathrm{CO}_{2}$ was given at the American Physical Society meeting, Washington, D. C. April 1964 [C. K. N. Patel, W. L. Faust, and R. A. McFarlane, Bull. Am. Phys. Soc. 9, 500 (1964)].

${ }^{4}$ C. K. N. Patel, W. R. Bennett, Jr., W. L. Faust, and R. A. McFarlane, Phys. Rev. Letters 9, 102 (1962).
}

tions seen in laser oscillation are even more appropriate, since the $\mathrm{CO}_{2}$ gas pressure used was typically 0.2 Torr (considerably lower than that used in the absorption measurements cited earlier). Thus, further highresolution spectroscopy should lead to a better determination of the transition frequencies and of the molecular constants. The rotational transitions seen in laser oscillation were identified to be from $P(12)$ to $P(38)$ for the $00^{\circ} 1-10^{\circ} 0$ vibrational band and from $P(22)$ to $P(34)$ for the $00^{\circ} 1-02^{\circ} 0$ vibrational band.

In the following section we will describe the experimental apparatus and techniques used in this investigation of stimulated emission spectrum of $\mathrm{CO}_{2}$ molecule. In Sec. III we will give the details of the experimental results and identification of the laser transitions (in accordance with Refs. 1 and 5). In the last section we will give a theoretical treatment and interpretation of the experimental results. The theoretical treatment satisfactorily explains the experimental findings, and especially the fact that no $R$-branch rotational transitions of the two vibrational bands $00^{\circ} 1-10^{\circ} 0$ and $00^{\circ} 1-02^{\circ} 0$ were seen in laser oscillation. The theoretical interpretation also leads to an interesting conclusion that for the rotational transitions between a pair of vibrational levels, because of their statistical weights, optical gain can exist for the $P$-branch transitions even when there is no net population inversion between the total population densities in the two vibrarional levels. And even though the treatment given in Sec. IV is specifically for $\mathrm{CO}_{2}$, it is also applicable to other linear polyatomic molecules like $\mathrm{CS}_{2}, \mathrm{~N}_{2} \mathrm{O}$, etc. as well as to diatomic molecules.

\section{EXPERIMENTAL TECHNIQUE}

The experimental setup used in the $\mathrm{CO}_{2}$ laser experiments consisted of a far-infrared optical maser similar to the one described in Ref. 6. The quartz discharge

${ }^{5}$ G. Herzberg, Molecular Spectra and Molecular Structure II, Infrared and Raman Spectra of Polyatomic Molecules (D. Van Nostrand Company, Inc., Princeton, New Jersey, 1945).

${ }^{6}$ W. L. Faust, R. A. McFarlane, C. K. N. Patel, and C. G. B. Garrett, Phys. Rev. 133, A1476 (1964). 
tube (see Fig. 1 of Ref. 6 ) was 25.4 mm i.d. and $5 \mathrm{~m}$ long. The optical resonator cavity was formed with a pair of near-confocal silicon mirrors, which were coated with vacuum-deposited aluminum for high reflectivity in the infrared. Coupling of energy out from the cavity was obtained by either (a) making the aluminum coating on one of the mirrors partially transparent or (b) leaving a small $(1.0 \mathrm{~mm}$ diam) area uncoated at the center of the output mirror. (The relative advantages of the two techniques have been discussed in Ref. 7). It was found that the second method was generally more satisfactory, and the results reported in this paper were obtained with that method. As such there was no additional wavelength-discriminating device (such as dielectric mirror coatings capable of giving high reflectivity in a narrow region of wavelengths) intentionally introduced in the optical cavity. Thus the long-wavelength limit of the laser tube was determined by diffraction losses, while the short-wavelength limit was set by the coupling of energy out through the aperture in one of the mirrors. The present tube dimensions gave a long-wavelength limit of about $35 \mu$ (corresponding to a diffraction loss of about $1 \%$ for the fundamental mode of the near-confocal optical cavity). At $9-10 \mu$, about $2 \%$ of the energy in the fundamental transverse mode is expected to be coupled out through the $1.0-\mathrm{mm}$ uncoated aperture in the silicon mirror. Discharge in $\mathrm{CO}_{2}$ was produced by using dc excitation. (In a limited number of cases, high current pulses of $1-\mu \mathrm{sec}$ duration were also used for investigation of the $\mathrm{CO}_{2}$ optical maser.)

A copper-doped germanium photoconductor at $4.2^{\circ} \mathrm{K}$ with N.E.P. $\approx 5 \times 10^{-11} \mathrm{~W} / \mathrm{cps}^{1 / 2}$ (Noise Equivalent Power) was used as the detector for the $9-10-\mu$ laser transitions. The speed of response of the detector was estimated to be faster than about $1 \mu \mathrm{sec}$, as estimated from looking at the rise time of the laser output at $7.6510 \mu$ when a helium-neon ${ }^{6}$ discharge was pulsed. Power measurements were carried out with a thermopile and were cross checked on the $\mathrm{Ge}: \mathrm{Cu}$ photoconductor from the data supplied by its manufacturer. Precise wavelength measurements for the $\mathrm{CO}_{2}$ laser transitions were carried out using a 1-m Jarrell-Ash scanning spectrometer equipped with a suitable grating. The wavelength measurements were judged to be accurate to better than $\pm 5 \AA$ on an absolute scale (on comparison with the known 3.39224- $\mu$ laser transition from a helium-neon discharge). The relative accuracy of the wavelength measurements is probably better than $\pm 2 \AA$. The spectrometer was evacuated during wavelength measurements. The light path between the spectrometer and the laser was filled with dry nitrogen to avoid troubles arising from water vapor and $\mathrm{CO}_{2}$ absorption in air.

${ }^{7}$ C. K. N. Patel, W. L. Faust, R. A. McFarlane, and C. G. B. Garrett, Appl. Phys. Letters 4, 18 (1964).

\section{RESULTS AND DISCUSSION}

Tables I and II give the wavelengths at which laser oscillation has been obtained. Table I lists the wavelengths which were identified to belong to the $P$-branch rotational transitions of the $00^{\circ} 1-10^{\circ} 0$ band of $\mathrm{CO}_{2}$.

TABLE I. cw laser oscillation wavelengths in the $00^{\circ} 1-10^{\circ} 0$ band of $\mathrm{CO}_{2}$.

\begin{tabular}{ccc}
\hline $\begin{array}{c}\text { Measured vacuum } \\
\text { wavelength }(\mu)\end{array}$ & $\begin{array}{c}\text { Frequency } \\
\left(\mathrm{cm}^{-1}\right)\end{array}$ & $\begin{array}{c}\text { Transition } \\
00^{\circ} 1-10^{\circ} 0 \text { band }\end{array}$ \\
\hline 10.5135 & 951.16 & $P(12)$ \\
10.5326 & 949.43 & $P(14)$ \\
10.5518 & 947.70 & $P(16)$ \\
10.5713 & 945.96 & $P(18)$ \\
10.5912 & 944.18 & $P(20)$ \\
10.6118 & 942.35 & $P(22)$ \\
$10.6324 \mathrm{a}$ & 940.52 & $P(24)$ \\
10.6534 & 938.67 & $P(26)$ \\
10.6748 & 936.78 & $P(28)$ \\
10.6965 & 934.88 & $P(30)$ \\
10.7194 & 932.89 & $P(32)$ \\
10.7415 & 930.96 & $P(34)$ \\
10.7648 & 928.95 & $P(36)$ \\
10.7880 & 926.95 & $P(38)$ \\
\hline \hline
\end{tabular}

a Strongest transition in the group; $P_{\text {out }} \approx 1 \mathrm{~mW}$.

TABLE II. cW laser oscillation wavelengths in the $00^{\circ} 1-02^{\circ} 0$ band of $\mathrm{CO}_{2}$.

\begin{tabular}{ccc}
\hline $\begin{array}{c}\text { Measured vacuum } \\
\text { wavelength }(\mu)\end{array}$ & $\begin{array}{c}\text { Frequency } \\
\left(\mathrm{cm}^{-1}\right)\end{array}$ & $\begin{array}{c}\text { Transition } \\
00^{\circ} 1-02^{\circ} 0 \text { band }\end{array}$ \\
\hline 9.5691 & 1045.03 & $P(22)$ \\
9.5862 & 1043.17 & $P(24)$ \\
9.6063 & 1041.28 & $P(26)$ \\
$9.6211^{\circ}$ & 1039.39 & $P(28)$ \\
9.6391 & 1037.44 & $P(30)$ \\
9.6576 & 1035.45 & $P(32)$ \\
9.6762 & 1033.47 & $P(34)$ \\
\hline
\end{tabular}

a Strongest transition in the group.

The wavelengths and the frequencies given are vacuum numbers. Table II gives similar description for the $00^{\circ} 1-12^{\circ} 0$ band oscillation wavelengths. It can be noticed that for both the bands, only $P$-branch transitions have been seen in laser action. The wavelengths measured are in slight disagreement with previous measurements of the transitions in absorption. ${ }^{1}$ Taking the values of $B_{000^{\circ}}=0.3866 \mathrm{~cm}^{-1}, B_{10}{ }^{\circ} 0=0.3897 \mathrm{~cm}^{-1}$, and $B_{02}{ }^{\circ}=0.3899 \mathrm{~cm}^{-1}$ as given by Herzberg, ${ }^{5}$ we find that the band-center frequencies are given in the following table:

$\begin{array}{cccc} & \text { Measured } & & \text { Measured } \\ \text { Frequencies }\left(\mathrm{cm}^{-1}\right) & \text { (this work) } & \text { Ref. } 8 & \text { (Ref. 9) } \\ \nu \text { band center } 00^{\circ} 1-10^{\circ} 0 & 960.94 & 960.9_{7} & 960.77 \\ \nu \text { band center } 00^{\circ} 1-02^{\circ} 0 & 1063.74 & 1063.7_{2} & 1063.57\end{array}$

These frequencies are in good agreement with the band-center frequencies as given in Ref. 8 . The differ-

${ }^{8}$ K. Rossmann, W. L. France, K. N. Rao, and H. H. Nielsen, J. Chem. Phys. 24, 1007 (1956); C. P. Courtoy, Ann. Soc. Sci. Bruxelles Ser. I 73, 5 (1959). 


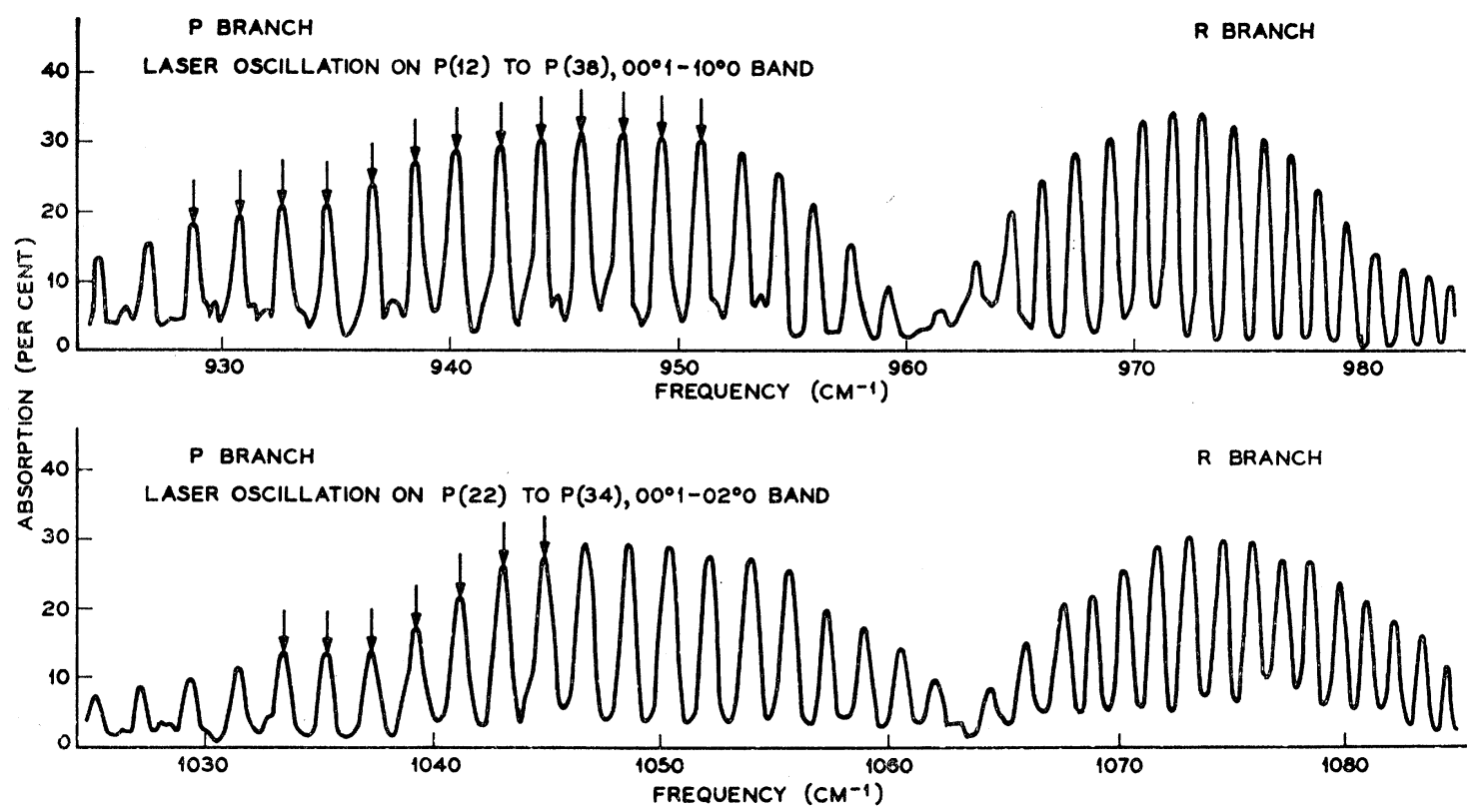

Fig. 1. Absorption spectrum for (a) $00^{\circ} 1-10^{\circ} 0$ band (top) and (b) for $00^{\circ} 1-02^{\circ} 0$ band (bottom) as measured by Barker and Adel in Ref. 1 . The frequency scale is corrected in both the cases to read vacuum wave numbers. The laser wavelengths are indicated by arrows on the particular transitions responsible.

ences in the band-center frequencies measured here and those given by Dennison ${ }^{9}$ (the frequencies given in Ref. 1 are measured in air, and Ref. 9 gives the vacuum wavenumbers) are believed to be real since the absolute accuracy of wavelength determination is better by a factor of three over the discrepancy. The difference of $0.17 \mathrm{~cm}^{-1}$ for the $00^{\circ} 1-10^{\circ} 0$ band and for the $00^{\circ} 1-02^{\circ} 0$ band may be the result of the pressure shift of the transition frequencies in measurements by Barker and Adel. ${ }^{1}$ With increase of pressure, the frequency shift is to be expected towards red..$^{10}$ This is what we find here. Barker and Adel's measurements were carried out at an atmospheric pressure of $\mathrm{CO}_{2}$ while our wavelength measurements for the laser transitions were made at $\mathrm{CO}_{2}$ pressure of 0.2 Torr. The effect of pressure broadening cannot be estimated in the measurements of Barker and Adel, but for some of the lines (the absorption spectrum is reproduced in Fig. 1 from Ref. 1) the width at half-absorption points seems to be in excess of their quoted instrument width of $0.5 \mathrm{~cm}^{-1}$. This, if real, points to a rather substantial line broadening. The Doppler width for the $\mathrm{CO}_{2}$ molecules at room temperature (in the experiment in Ref. 1) can be calculated to be $0.002 \mathrm{~cm}^{-1}$. The natural linewidth for these transitions is expected to be generally smaller, and thus if the linewidth as seen from Fig. 1 is really greater than the instrumental width, the Holtzmark broadening is playing an important role in determining the width of the absorption lines.

${ }^{9}$ D. M. Dennison, Rev. Mod. Phys. 12, 175 (1940).

${ }^{10}$ A. C. G. Mitchell and M. W. Zemansky, Resonance Radiation and Excited Atoms (Cambridge University Press, New York, 1961).
The strongest laser transition in the $00^{\circ} 1-10^{\circ} 0$ band occurs at $10.6324 \mu$, and the power output was about $1 \mathrm{~mW}$ on a $\mathrm{cw}$ basis. The strongest transition in the $00^{\circ} 1-02^{\circ} 0$ band occurred at $9.6211 \mu$ and the power output was slightly less than that at $10.6324 \mu$. The optical gains at these two wavelengths were estimated to be about $3 \% /$ meter and $2 \%$ /meter, respectively. Under pulsed operation with current pulses of about 1- $\mu \mathrm{sec}$ duration, the peak output power increased by about a factor of 10. However, neither under CW nor under pulsed conditions was saturation of laser power output possible with respect to the electrical power input to the discharge.

Figure 2 is an energy-level diagram ${ }^{5}$ of $\mathrm{CO}_{2}$ showing the pertinent vibrational levels. (Courtoy ${ }^{8}$ gives recent determinations of the levels. These differ slightly from the values shown in Fig. 2.) The rotational levels are not shown on the diagram for the sake of simplicity. The upper level for both the bands which is $\Sigma_{u}+\left(00^{\circ} 1\right)$ is connected to the ground state $\Sigma_{g}{ }^{+}\left(00^{\circ} 0\right)$ through the strongly allowed optical transitions at $2349.3 \mathrm{~cm}^{-1}$. The lower laser levels $\Sigma_{g}+\left(10^{\circ} 0\right.$ and $\left.02^{\circ} 0\right)$ both decay to the $\Pi_{u}\left(01^{1} 0\right)$ through radiative transitions which are reported to be medium in Ref. 5 . The $\Pi_{u}\left(01^{1} 0\right)$ levels are connected to the $\mathrm{CO}_{2}$ ground state through very strong optically allowed transitions at $667.3 \mathrm{~cm}^{-1}$. Thus the laser scheme works like a four-level system. Again the alternate lines in the rotational spectrum of $\Sigma_{u}{ }^{+}-\Sigma_{g}{ }^{+}$band of $\mathrm{CO}_{2}$ are missing because $\mathrm{CO}_{2}$ is a linear and symmetric molecule for which symmetry considerations result into the absence of odd $J$ levels in the $\Sigma_{\theta}{ }^{+}$vibrational levels and of even $J$ levels in the 


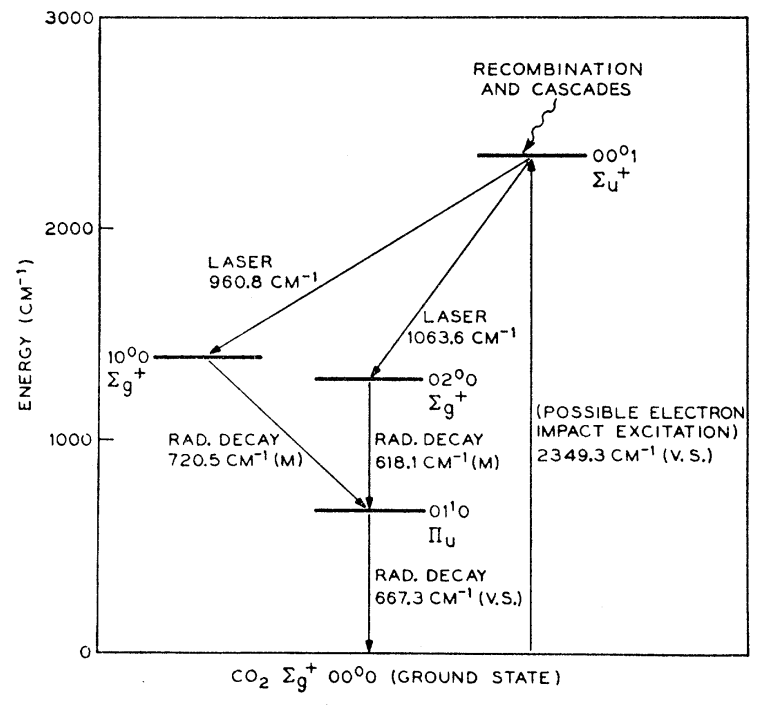

FIG. 2. Pertinent parts of the energy-level diagram showing the laser transitions.

$\Sigma_{u}{ }^{+}$vibrational levels. Since $l$ is zero for both $\Sigma_{u}{ }^{+}$and $\Sigma_{g}{ }^{+}$, the symmetry considerations require that $\Delta J=0$ transitions be not allowed and hence the $Q$ branch is missing. The $P$-branch rotational transitions lie on the long-wavelength side of the band center while the $R$ branch transitions lie on the short-wavelength side of the band center. $R$-branch rotational transitions have not been seen in laser oscillation for both the $00^{\circ} 1-10^{\circ} 0$ and the $00^{\circ} 1-02^{\circ} 0$ bands. (See Fig. 1 for the $P$ - and $R$-branch rotational transitions for the $00^{\circ} 1-10^{\circ} 0$ and $00^{\circ} 1-02^{\circ} 0$ bands as seen in absorption by Barker and Adel in Ref. 1.)

The excitation of molecules to the upper laser levels may be through electron impact excitation from the ground state, from recombinations, or from cascades from levels above. A time-dependence study of the laser power output under $1-\mu$ sec pulsed-discharge conditions revealed that probably the processes involved are the last two. Figure 3 shows the typical results of such a time-dependence study of laser output. The top two traces in Fig. 3(a) show the pulse voltage and current waveforms in the laser tube, respectively. The current pulse is nominally $1 \mu \mathrm{sec}$ long and shows pronounced ringing effects due to mismatch in the current viewing circuit. The peak pulse voltage and the peak pulse current are $15 \mathrm{kV}$ and $15 \mathrm{~A}$, respectively. The bottom trace in Fig. 2(a) shows the light output at $4830 \AA$ Arom the side of the discharge tube. A low-resolution grating monochromator was used to isolate the particular line. The 4830- $\AA$ line corresponds to the band head of the $0-1$ band of $B^{1} \Sigma-A^{1} \Pi$ angstrom band (electronic band) of $\mathrm{CO}$. A fast photomultiplier was used as the detector. It can be easily seen that there is very small delay between the current pulse and light output pulse at $4830 \AA$. This was seen to be true of other bands of CO system. The $4830-\AA$ band was chosen because of the best signal-to-noise ratio available with the photomultiplier 6199. (This very small delay is consistent with earlier report of laser oscillation on angstrom-band transitions in a pulsed $\mathrm{CO}$ discharge. ${ }^{11}$ ) Thus the excitation of $\mathrm{CO}$ bands in a $\mathrm{CO}_{2}$ discharge involves a very small delay from the pulse current. Figure 3(b) shows (in the top trace) the laser output at $10.6324 \mu$, and the lower two traces show the 4830 -Å light-output pulse and current pulse, respectively. Note that the time scale has been now changed to $50 \mu \mathrm{sec} / \mathrm{cm}$. The most striking feature is that the laser output pulse lags the excitation current pulse by a period of over $300 \mu \mathrm{sec}$. The laser output starts then and builds up quite rapidly (rise time $\approx 5$ $\mu \mathrm{sec})$. The laser oscillation under a $1-\mu \mathrm{sec}$ current-pulse

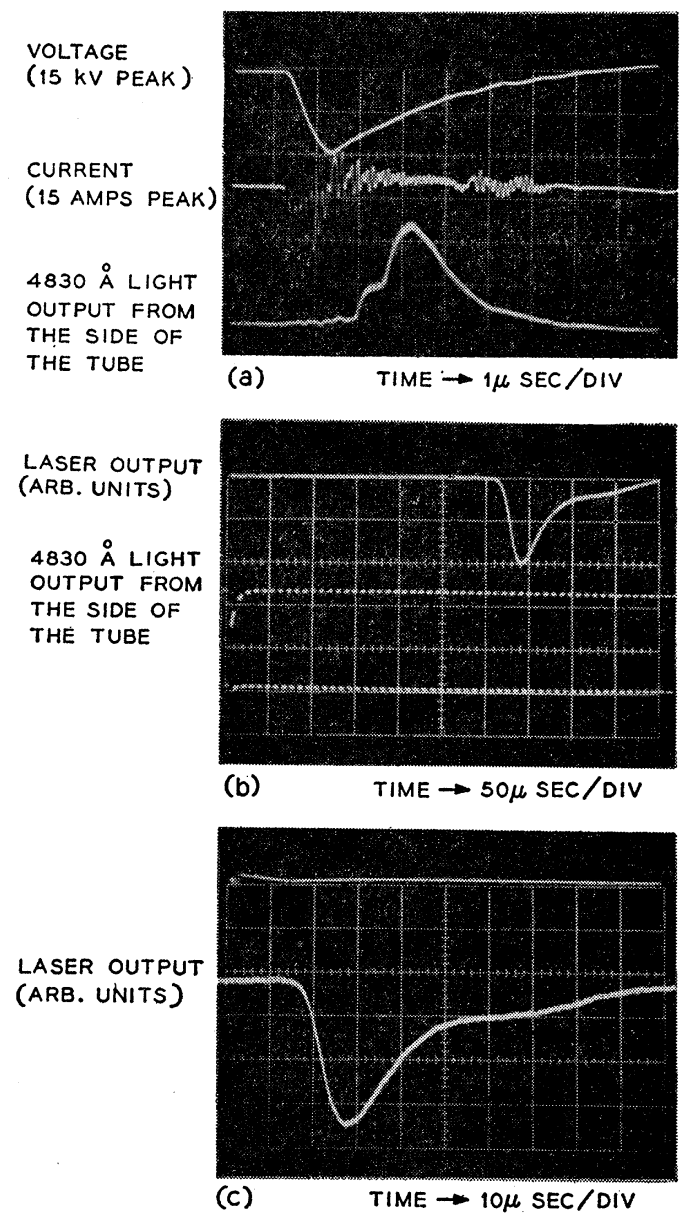

FIG. 3. Behavior of the $\mathrm{CO}_{2}$ laser at $10.6324 \mu$ under pulsed discharge conditions; (a) top trace: pulse voltage (15-kV peak); middle trace: pulse current (15-A peak); bottom trace: $4830-\AA$ light output (CO $B^{1} \Sigma \rightarrow A^{1} \Pi$ band) from the side of discharge tube. Time scale $=1 \mu \mathrm{sec} /$ division for all three traces. (b) top trace: laser output at $10.6324 \mu$; middle trace: $4830-\AA$ - light output from the side; bottom trace: current pulse at far left. [Note the long $(\sim 300 \mu \mathrm{sec})$ time delay between the current pulse and the commencement of laser oscillation.] Time scale: $50 \mu \mathrm{sec} /$ division for both; (c) laser output display on a $10-\mu \mathrm{sec} /$ division time scale showing long decay time.

${ }^{11}$ L. E. S. Mathias and J. T. Parker, Phys. Letters 7, 194 (1964). 
excitation lasts for about $80 \mu \mathrm{sec}$. The decay of the laser output is far from being a single exponential, as seen in Fig. 2(c) with the time scale expanded to 10 $\mu \mathrm{sec} /$ division. At least two distinct decay mechanisms seem to be operative. One of them is seen to have a decay time of $\sim 50 \mu \mathrm{sec}$ and the other $\sim 150 \mu \mathrm{sec}$. The response time of the $\mathrm{Cu}: \mathrm{Ge}$ photoconductor used for detection of the 9.4- $\mu$ and the 10.4- $\mu$ laser radiation was considerably shorter (see Sec. II). The laser output on other transitions listed in Tables I and II showed the same general behavior.

From these time dependence results, we can now make some remarks about the processes responsible for creation of population inversion between the $00^{\circ} 1$ and the $10^{\circ} 0$ and $02^{\circ} 0$ levels. In a low-pressure discharge, the following are a few of the excitation processes which may be operative:

$$
\begin{gathered}
\mathrm{CO}_{2}+(e+\mathrm{KE}) \rightarrow \mathrm{CO}^{*}+\mathrm{O}+e \\
\rightarrow \mathrm{CO}+\mathrm{O}^{*}+e .
\end{gathered}
$$

(The asterisks imply excited states.)

$$
\begin{aligned}
\mathrm{CO}+(e+\mathrm{KE}) & \rightarrow \mathrm{CO}^{*}+e \\
\mathrm{CO}^{*} & \rightarrow \mathrm{CO}^{* *}+h \nu .
\end{aligned}
$$

The processes in Eqs. (1), (2), and (3) involve a very small delay and are primarily responsible for the visible light output (in the $\mathrm{CO}$ band system) from the side of the tube. The next step in excitation process involves recombination and a possible cause for the long delay time for starting of oscillation on the 9.4 and 10.4- $\mu \mathrm{CO}_{2}$ laser transitions. (Some of the following reactions have been suggested ${ }^{12}$ also to explain the emission from low-pressure $\mathrm{O}+\mathrm{CO}$ flames. In addition, Gaydon $^{12}$ suggests that the afterglow emission of $\mathrm{CO}$ flame bands observed from a low-pressure $\mathrm{CO}_{2}$ discharge may be considered a sort of flame emission due to recombination of the dissociation products, $\mathrm{O}+\mathrm{CO}$, rather than direct excitation of the $\mathrm{CO}_{2}$ molecules.)

and/or

$$
\mathrm{CO}\left(X^{1} \Sigma^{+}\right)+\mathrm{O}\left({ }^{3} P\right) \rightarrow \mathrm{CO}_{2}{ }^{*}
$$

$$
\rightarrow \mathrm{CO}_{2}\left(00^{\circ} 1\right)+h \nu \text {. }
$$

$\left[\mathrm{CO}_{2} *\right.$ in Eq. (4a) has to be a triplet state to conserve spin.] $\mathrm{CO}_{2}{ }^{*}$ in Eq. (4a) then makes a radiationless transition to the ${ }^{1} B$ electronic state. ${ }^{12}$

and

$$
\mathrm{CO}_{2}{ }^{*} \rightarrow \mathrm{CO}_{2}{ }^{* *}\left({ }^{1} \mathrm{~B}\right)
$$

$$
\mathrm{CO}_{2} * *\left({ }^{1} B\right) \rightarrow \mathrm{CO}_{2}\left({ }^{1} \Sigma^{+}\right)+h \nu .
$$

In the present work it is not possible to distinguish between the process in Eq. (4b) and the combination of processes in Eqs. (4a) and (5). Thus the time delay of

12 A. G. Gaydon, The Spectroscopy of Flames (Chapman and Hall, London, 1957); M. A. A. Clyne and B. A. Thrush, Proc. Royal Soc. (London) 269, 404 (1962).
$300 \mu$ sec seen in Fig. 2(b) may be due to (a) recombination delay involved in processes in Eqs. (4a) and (4b), and/or (b) time involved in cascade process in Eq. (5). It is not entirely impossible that the selective excitation described in Eq. (4b) may not be the dominant one.

The process of direct excitation of $\mathrm{CO}_{2}$ molecules to the $00^{\circ} 1$ levels by electron impact upon the ground state of the molecule is not ruled out. As mentioned earlier, the $00^{\circ} 1$ level is connected to the ground state through strongly allowed transitions at $2349 \mathrm{~cm}^{-1}$ (see Fig. 2). Thus, the excitation process

$$
\mathrm{CO}_{2}+(e+\mathrm{KE}) \rightarrow \mathrm{CO}_{2}\left(00^{\circ} 1\right)+e
$$

can have large cross section. However, if the direct electron-impact excitation were the dominant one, a delay of $300 \mu \mathrm{sec}$ between the current pulse and the laser output pulse seems rather unreasonable to expect. To explain the 300- $\mu$ sec delay seen in Fig. 3(b), if the electron-impact excitation were a dominant process in populating the laser levels, we would be required to accept that under pulsed-current excitation conditions, both the upper and the lower laser levels are simultaneously populated by electron impact. And even though the excitation lasts for only about $1 \mu \mathrm{sec}$, no inversion is achieved during that period. The lower laser levels decay with lifetimes shorter than the lifetimes for the upper laser levels. (This is required ${ }^{13}$ and is probably satisfied since the laser action is achieved under $\mathrm{cw}$ discharge conditions also.) With lower laser level, lifetimes of the order of $100 \mu \mathrm{sec}$ and longer lifetimes for the upper laser levels, it can be seen that even with the upper and the lower laser levels equally populated to have no inversion during the excitation current pulse, population inversion will be established after a delay of several hundred $\mu \mathrm{sec}$ and laser oscillation would commence. The strongest objection, though, for accepting this explanation does not lie in accepting the long lifetimes for the laser levels (which may very well be true for the vibrational-rotational levels in the ground electronic state), but in accepting that both the upper and lower laser levels are populated during the current pulse so that no laser action is possible. The lower laser level, having the same parity as that of the ground state of $\mathrm{CO}_{2}$, is not optically connected to the ground state. Thus, the reaction

$$
\mathrm{CO}_{2}+(e+\mathrm{KE}) \rightarrow \mathrm{CO}_{2}\left(10^{\circ} 0 \text { or } 02^{\circ} 0\right)+e
$$

cannot be expected to have a cross section comparable to that for the excitation of the upper laser level $\left(00^{\circ} 1\right)$ through the reaction given in Eq. (6). Hence, the explanation that equal populations in the upper and the lower laser levels during current pulse is responsible for the long delay time between the current pulse and the laser pulse is difficult to accept.

On the other hand, the long delay can be easily

${ }^{13}$ W. R. Bennett, Jr., Appl. Optics Suppl., 24, (1962). 
attributed to the recombination and cascade processes in Eqs. (4) and (5). The long decay time of about $80 \mu \mathrm{sec}$ [as seen from Fig. 3(c)] is then accounted for by the long lifetime of the species of atoms/molecules which are responsible for populating the upper laser level. Here it will be the lifetime of the $\mathrm{CO}$ and $\mathrm{O}$ species in the afterglow of a low puressure pulsed discharge. $\mathrm{CO}$ and $\mathrm{O}$ recombine as described in Eqs. (4a) and (4b) and form a rather long-lived supply rate or excitation rate for supplying the molecules to the upper laser level. The time-dependence behavior of the power output for the $\mathrm{CO}_{2}$ laser is somewhat similar to that for the 1.1523- $\mu$ power output in a helium-neon laser. ${ }^{14}$ (In this case, under pulse excitation, the laser output in the afterglow period follows the decay time of the $2^{3} S_{1}$ metastable state of helium which is responsible for selectively populating the upper laser level in neon.) Thus, from the analysis of the time-dependence studies, we prefer to conclude that the dominant process leading excitation of $\mathrm{CO}_{2}$ molecules to the $00^{\circ} 1$ level is recombination and/or cascade, rather then direct electron-impact excitation from the ground state of $\mathrm{CO}_{2}$.

\section{THEORETICAL INTERPRETATION}

In Ref. 15, a theoretical analysis of the $\mathrm{CO}_{2}$ laser data was given based on a simple model. The analysis explained the experimental findings satisfactorily. We will give here the generalized expressions for optical gain and make a few additional remarks. (See also also Ref. 16 for some general remarks and a proposal for laser action on vibrational-rotation transitions.)

$$
\text { optical gain }=\exp \left(\alpha_{12} l\right)
$$

where $l=$ length of the medium and $\alpha_{12}=$ gain (or absorption) coefficient of the medium for a transition between levels 1 and 2. From Mitchell and Zemansky ${ }^{10}$ (and also Bennett ${ }^{13}$ )

$$
\alpha_{12}=\left(\frac{\ln 2}{\pi}\right)^{1 / 2} \frac{g_{1} A_{12}}{4 \pi}\left(\frac{N_{1}}{g_{1}}-\frac{N_{2}}{g_{2}}\right) \frac{\lambda_{12}^{2}}{\Delta \nu_{D}},
$$

where $N_{1}=$ population density in the upper laser level; $N_{2}=$ population density in the lower laser level; $\lambda_{12}=$ wavelength of the transition; $g$ 's are the statistical weights of levels 1 and $2 ; \Delta \nu_{D}=$ Doppler width of the transition. This formula assumes that the transition is predominantly Doppler-broadened. Now assuming a simplified model of a vibrational level in which the rotational level populations are described by a Boltzmann distribution at temperature $T_{\text {rot }}$, it can be seen from Ref. 5 that

$$
N_{J}=\left(N / Q_{r}\right) g_{J} \exp \left[-F(J) h c / k T_{\text {rot }}\right]
$$

${ }^{14}$ A. Javan, W. R. Bennett, Jr., and D. R. Herriott, Phys. Rev. Letters 6, 106 (1961).

${ }^{15}$ C. K. N. Patel, Phys. Rev. Letters 12, 588 (1964).

${ }^{16}$ J. C. Polanyi, J. Chem. Phys. 34, 347 (1961). where $N_{J}=$ population density in $J$ th rotational level; $N=\Sigma_{J} N_{J} ; Q_{r}=$ rotational partition function (see Ref. 5); $h=$ Planck's constant; $c=$ velocity of light; $k$ =Boltzmann's constant; and $F(J)=$ energy of $J$ th rotational level from the 0 th rotational level, i.e.

with

$$
F(J)=B J(J+1)-D J^{2}(J+1)^{2}
$$

$$
D \ll B \text {. }
$$

For linear polyatomic molecules (and also for diatomic molecules),

$$
Q_{r}=\sum_{J}(2 J+1) e^{-F(J) h c / k T} .
$$

For small $B$ and large $T$

$$
Q_{r} \approx k T / h c B \text {. }
$$

Thus, for linear polyatomic (and diatomic) molecules

Now

$$
N_{J}=N(h c B / k T) g_{J} e^{-F(J) h c / k T} .
$$

$$
A_{I_{J}{ }^{2} J_{ \pm}}=\left(64 \pi^{4} \nu^{3}{ }_{{ }_{1 J}{ }^{2} J_{ \pm 1}} / 3 h_{J}\right) / \Sigma\left|R^{1 J^{2} J \pm 1}\right|^{2}
$$

where $\nu_{1 J^{2} J_{ \pm 1}}=$ frequency of transition from $J$ th rotational level in vibrational level 1 (upper level) to $(J \pm 1)$ th rotational level in vibrational level 2 (lower level).

$$
\begin{aligned}
\Sigma\left|R^{1 J^{2} J \pm 1}\right|^{2} & =\text { matrix element for the transition } \\
& =K_{12} S_{J}
\end{aligned}
$$

where $K_{12}=J$ independent part of the matrix element; $S_{J}=J$-dependent part of the matrix element; and

$$
\Delta \nu_{D}=\left(2 / \lambda_{1_{J} 2_{J \pm 1}}\right)\left[\left(2 k T_{\mathrm{mol}} / M\right) \ln 2\right]^{1 / 2},
$$

where $\lambda_{1_{J} J_{ \pm 1}}=$ wavelength of the transition, $T_{\text {mol }}$ $=$ molecular temperature, and $M=$ molecular mass. Substituting Eqs. (13), (14), (15), and (16) into Eq. (9), we obtain

$$
\begin{array}{r}
\alpha_{1 J J_{ \pm 1}}=\frac{8 \pi^{3} c^{4} k_{12}}{3 k T_{\mathrm{rot}}\left(2 \pi k T_{\mathrm{mol}} / M\right)^{1 / 2}} S_{J}\left[N_{1} B_{1} e^{-F_{1}(J) h c / k T}\right. \\
\left.-N_{2} B_{2} e^{-F_{2}(J \pm 1) h c / k T}\right] .
\end{array}
$$

Now

$$
S_{J}=J+1 \text { for } P \text {-branch rotational transitions }
$$
and

$$
S_{J}=J \text { for } R \text {-branch rotational transitions, }
$$

where $J$ is the rotational quantum number of the upper level. With simplifying assumption $T_{\text {mol }} \approx T_{\text {rot }} \approx T$ we obtain the gain coefficient expressions given in Ref. 15 .

(a) For $P$-branch transitions, i.e., for $P(J+1)$ transitions

$\alpha_{1 J 2 J+1}=\frac{8 \pi^{3} c^{4} K_{12}}{3 k T(2 \pi k T / M)^{1 / 2}}(J+1)\left(N_{1} B_{1} e^{-F_{1}(J) h c / k T}\right.$
$\left.-N_{2} B_{2} e^{-F_{2}(J+1) h c / k T}\right)$. 
(b) For $R$-branch transitions, i.e., for $R(J-1)$ transitions

$$
\begin{gathered}
\alpha_{1 J 2 J-1}=\frac{8 \pi^{3} c^{4} K_{12}}{3 k T(2 \pi k T / M)^{1 / 2}} J\left(N_{1} B_{1} e^{-F_{1}(J) h c / k T}\right. \\
\left.-N_{2} B_{2} e^{-F_{2}(J-1) h c / k T}\right) .
\end{gathered}
$$

It should be pointed out that the expressions given in Eqs. (20) and (21) are quite general, and should be applicable to all diatomic and linear polyatomicmolecule gas low-pressure discharge situations. Also in case $\alpha$ 's are negative, the expression for gain given in Eq. (8) implies a loss.

In Ref. 15, gain coefficients were evaluated for the $00^{\circ} 1-10^{\circ} 0$ and the $00^{\circ} 1-02^{\circ} 0$ vibrational-rotational bands of $\mathrm{CO}_{2}$. We will not repeat the conclusions reached in Ref. 15 but will leave it to the reader for reference. We will, however, make some additional remarks about fitting the exprerimental data to the theoretical model. After having obtained gain coefficient as a function of upper level $J$ for various temperatures and for various upper-to-lower laser-level population ratios, to determine the best fit, the following criteria were used in the case of $00^{\circ} 1-10^{\circ} 0$ and $00^{\circ} 1-02^{\circ} 0$ bands of $\mathrm{CO}_{2}$.

(1) For each of the bands, the two extreme oscillating transitions $\left(J=11\right.$ and $J=37$ for $00^{\circ} 1-10^{\circ} 0$ band and $J=21$ and $J=33$ for $00^{\circ} 1-02^{\circ} 1$ band) should have the same optical gain.

(2) The peak of the gain curve as obtained from expressions in Eqs. (20) and (21) should coincide with the strongest experimentally observed laser transition.

The above two comparisons can be made on the normalized gain plots as given in Fig. 2, Ref. 15.

(3) The threshold gain for oscillation for the $00^{\circ} 1-$ $10^{\circ} 0$ and $00^{\circ} 1-02^{\circ} 0$ bands (not normalized gain) should be in ratio of the single-pass loss in the laser for that particular wavelength. In the $\mathrm{CO}_{2}$ laser experiments it was estimated that the threshold gains for the $00^{\circ} 1-10^{\circ} 0$ and $00^{\circ} 1-02^{\circ} 0$ bands should be in the ratio of $1: 1.1$.

(4) The peak gains for $00^{\circ} 1-10^{\circ} 0$ and $00^{\circ} 1-02^{\circ} 0$ bands (not normalized gains) were measured to be in the ratio of 1.5:1. With the help of conditions (3) and (4) we can eliminate the unknown $K_{12}$ for the two bands by plotting the ratios of peak normalized gains to threshold normalized gains.

(5) The two bands should correspond to the same temperature $T$.

It is a simple exercise in eliminating the variables to see that, in the case of $\mathrm{CO}_{2} 00^{\circ} 1-10^{\circ} 0$ and $00^{\circ} 1-02^{\circ} 0$ bands, the simultaneous satisfaction of conditions (1) through (5) results in a unique determination of temperature $T$ and the relative population densities $N_{00}^{\circ}, N_{10}{ }^{\circ}$, and $N_{02}{ }^{\circ}$.

A computer programmed to satisfy these conditions with experimental findings yielded

$$
T=400^{\circ} \mathrm{K}, \quad N_{000^{\circ}}=N_{02^{\circ} 0}=1.05 N_{10^{\circ} 0} .
$$

The factor omitted from the analysis is the competition effects between $00^{\circ} 1-10^{\circ} 0$ and $00^{\circ} 1-02^{\circ} 0$ transitions originating in the same upper $00^{\circ} 1$ rotational levels.

In Ref. 15 a detailed argument was given as to why the $R$-branch transitions do not oscillate and this will not be repeated here, although it will suffice to say here that the experimental findings are satisfactorily explained by the theoretical interpretation given in Ref. 15.

\section{ACKNOWLEDGMENTS}

The author would like to thank D. MCNair for supplying the hot cathodes used in the experiments, Mrs. C. Lambert for the aid in computation, and R. J. Kerl for technical assistance. 


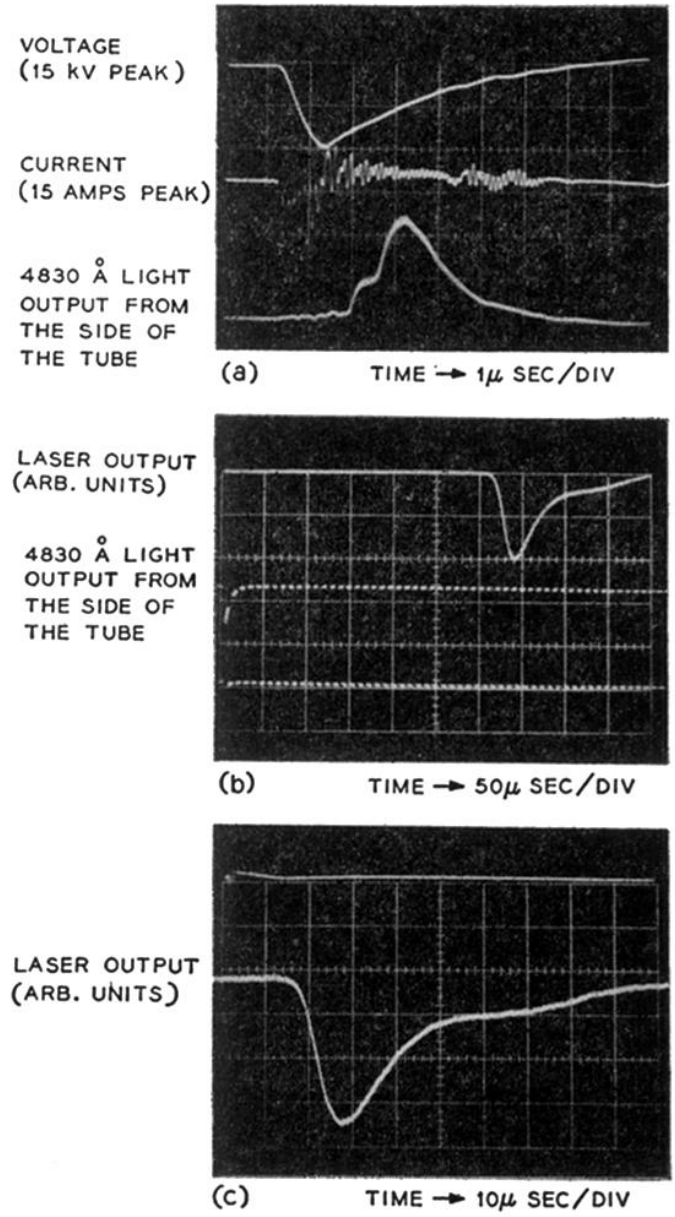

Fig. 3. Behavior of the $\mathrm{CO}_{2}$ laser at $10.6324 \mu$ under pulsed discharge conditions; (a) top trace: pulse voltage (15-kV peak); middle trace: pulse current (15-A peak); bottom trace: $4830-\AA$ light output (CO $B^{1} \Sigma \rightarrow A^{1} \Pi$ band) from the side of discharge tube. Time scale $=1 \mu \mathrm{sec} /$ division for all three traces. (b) top trace: laser output at $10.6324 \mu$; middle trace: $4830-\AA$ light output from the side; bottom trace: current pulse at far left. [Note the long $(\sim 300 \mu \mathrm{sec})$ time delay between the current pulse and the commencement of laser oscillation. T Time scale: $50 \mu \mathrm{sec} /$ division for both; (c) laser output display on a $10-\mu \mathrm{sec} /$ division time scale showing long decay time. 